\title{
Time and Location-Critical Emergency Message Dissemination for Vehicular Ad-Hoc Networks
}

\author{
Yanyan Zhuang, Jianping Pan, Yuanqian Luo, and Lin Cai
}

\begin{abstract}
One promise of Vehicular Ad-hoc Networks (VANET) is to considerably increase road safety and travel comfort by enabling inter-vehicle communications. Among a vast array of potential applications, emergency message (EM) dissemination has attracted a lot of attention in the literature. In this paper, we propose a time/location-critical (TLC) framework for EM dissemination and use our scalable modulation and coding (SMC) scheme to achieve the goal. In specific, vehicles near the accident site (or the point-of-interest location) receive guaranteed, detailed messages to take proper reaction immediately (e.g., slow down or change lanes), and vehicles further away have a high probability to be informed and make location-aware decisions accordingly (e.g., detour or reroute), with the assistance of reverse traffic when possible and necessary. The efficacy of the proposed framework is analyzed and validated by extensive numerical and simulation results. The TLC framework and the use of the SMC scheme are shown to be able to disseminate EMs effectively and efficiently by taking both the time and location criticality into account, while simplifying the design of radio transceivers and media access control protocols for VANET.
\end{abstract}

Index Terms - Vehicular ad-hoc networks, emergency message dissemination, scalable modulation and coding

\section{INTRODUCTION}

$\mathbf{T}$ HE ALLOCATION of a $75 \mathrm{MHz}$ licensed band at $5.9 \mathrm{GHz}$ for Dedicated Short Range Communications (DSRC) [1] has renewed interest from both industry and academia in Vehicular Ad-hoc Networks (VANET) to considerably increase road safety and travel comfort, which is also an important part for future Intelligent Transportation Systems (ITS) [2]. It is expected that ITS will bring huge economic and social impacts to our more and more connected lifestyles and activities, by enabling inter-vehicle communications with or without the assistance of roadside infrastructures.

Among the vast array of potential ITS applications, emergency message (EM) dissemination is considered to be very important. Many Media Access Control (MAC) and routing schemes have been proposed in the literature to facilitate EM dissemination in VANET [3]-[7]. When an accident happens or a certain road condition is observed (referred to as "pointof-interest", or POI, in this paper), the vehicles nearby need detailed information immediately to react properly (e.g., slow down or change lanes), due to the short distance to the POI and thus short reaction time. At the same time such information, likely with different levels of detail, needs to be disseminated further to allow following vehicles at different distances to make location-aware decisions accordingly (e.g., detour or reroute when possible). It is obvious that the time and location

Manuscript received 5 January 2010; revised 7 May 2010 and 12 July 2010. The authors are with the University of Victoria, Victoria, BC, Canada (email: \{yyzhuang,pan,yqluo,cai\}@uvic.ca).

Digital Object Identifier 10.1109/JSAC.2011.110118. criticality of EM dissemination should be taken into account at the same time.

Existing schemes, such as IEEE 802.11p [8], achieve message dissemination at different distances in different details by using different channels. Each of these channels has different transmission power limit and thus different transmission range and achievable data rate. The multi-channel operation ideally requires multiple radio transceivers, which unfortunately increase the system cost and complexity, thus impeding the initial development and deployment. Therefore, single-radio multi-channel (SRMC) operation is a more feasible solution. However, SRMC imposes some additional requirements such as node synchronization, and nodes have to return to the common Control Channel $(\mathrm{CCH})$ at the same time periodically. Switching to different channels at different central frequencies also adds extra timing overhead to stabilize transceivers, thus delaying the progress of EM dissemination.

Broadcast has many applications in VANET and also creates new challenges. In this paper, we propose a new time/locationcritical (TLC) framework for EM dissemination and use the scalable modulation and coding (SMC) scheme proposed by us in [9] to achieve the goal. By redefining the bit-to-symbol mapping in the modulation constellation, SMC allows us to encode information of different importance at the same time. With carefully designed mappings, nodes closer to the transmitter and with higher signal-to-interference-and-noiseratio (SINR) can decode more information of both high and low importance (i.e., more detailed information). On the other hand, nodes further away or with lower SINR decode less information from the same broadcast transmission. The SMC scheme fits very well with the TLC framework for EM broadcast, as nearby vehicles (often with high SINR) need guaranteed, detailed information for quick maneuvering (e.g., cruise control or even autopilot), while further-away vehicles can have an early warning first and then get more detailed information as they approach the POI.

Besides using SMC in the TLC framework, this paper also has the following contributions. We first obtain the moments of the size of the connected vehicle clusters accurately with a simple approach and give a close approximation to its distribution, by exploring the i.i.d. property of inter-vehicle distances revealed by the latest highway traffic measurement studies [12]. Second, we analyze how to use reverse traffic (the vehicles in the opposite direction, often available for highway traffic but possibly at different density and reliability) opportunistically to further extend the cluster size and meet the requirement of the TLC framework. We also derive the probability that further-away vehicles may miss the next detour exit due to the "carry-and-forward" delay introduced 
by reverse traffic. Third, the TLC framework and performance analysis have been validated by extensive simulation results, showing the efficacy of the framework and the analysis.

The rest of this paper is organized as follows. In Section II, we briefly overview VANET and review the related work in EM dissemination, as well as the latest traffic measurement results. We then present the TLC framework and show how the SMC scheme fits with the TLC framework in Section III. The cluster size, the benefit of using reverse traffic to further extend the cluster size, and the probability for vehicles to miss their detour deadline are obtained analytically in Section IV, each followed by the performance evaluation results. Section V concludes the paper with future work.

\section{BACKGROUND AND RELATED WORK}

\section{A. VANET and DSRC}

Compared with other forms of Mobile Ad-hoc Networks (MANET), VANET has its unique features. First, VANET has to support high mobility nodes, such as vehicles traveling on highways or in cities. Second, due to road constraints, its mobility is highly regular and predictable in normal conditions. There are new protocol stacks, from the physical layer to the application layer, proposed and standardized for DSRC [1] and VANET. Different countries may have different standards, and here we use IEEE 802.11p [8] as an example.

The $75 \mathrm{MHz}$ DSRC frequency band allocated by FCC has seven $10 \mathrm{MHz}$ channels (the first $5 \mathrm{MHz}$ band is reserved for future use) to minimize the inter-symbol interference over long distances (up to $1 \mathrm{~km}$ ) and with high mobility (over $120 \mathrm{~km} / \mathrm{h}$ ). The first and seventh channels are dedicated for high availability low latency (HALL) and intersection applications, respectively. The middle (fourth) channel is for control purposes with variable transmission power and a node is not allowed to occupy the channel longer than $200 \mu \mathrm{s}$ at a time. The second and third channels are for medium-range services (with transmission power limit $33 \mathrm{dBm}$ ), and can be bonded into a single $20 \mathrm{MHz}$ channel. The fifth and sixth channels (can be bonded as well) are for short-range services (with transmission power limit $23 \mathrm{dBm}$ ).

IEEE $802.11 \mathrm{p}$ reuses the orthogonal frequency-division multiplexing (OFDM) physical layer (PHY) of IEEE 802.11a, but with a $10 \mathrm{MHz}$ channel bandwidth, thus many OFDM timing parameters being scaled accordingly. IEEE $802.11 \mathrm{p}$ also adopts the IEEE 802.11e Enhanced Distributed Channel Access (EDCA) function with prioritized media access, matching the priority nature of VANET applications, but the contention resolution parameters can be different from IEEE 802.11e. Together with the frequency allocation scheme mentioned above, IEEE $802.11 \mathrm{p}$ achieves data rates of $3 \mathrm{Mbps}$ up to $1 \mathrm{~km}$ and $27 \mathrm{Mbps}$ within $200 \mathrm{~m}$ (or $6 \mathrm{Mbps}$ and $54 \mathrm{Mbps}$ if channel bonding is used), by using different transmission power and modulation schemes in different channels. Ideally, this multi-channel operation can be achieved by multiple transceivers, but such a design will increase system cost and complexity and impede initial deployment. Therefore, channel hopping with a single transceiver (e.g., SRMC) is more appealing. However, channel hopping not only requires node synchronization to return to the control channel at the same time (at most every $100 \mathrm{~ms}$ ), but also introduces additional delay to repeatedly stabilize transceivers at new frequencies. This motivates the single-radio single-channel (SRSC) design advocated in this paper, which can considerably simplify MAC protocols when compared with SRMC.

\section{B. EM Dissemination and VANET Connectivity}

Emergency message (EM) dissemination has attracted a lot of attention in the literature, and many MAC and routing protocols are proposed for VANET [3]-[7]. Due to the limited reaction time, EM normally should be broadcast to vehicles near the POI immediately and periodically; otherwise, the event may trigger chain reaction (e.g., vehicle pileup). Furthermore, the message with different levels of detail should be propagated (possibly by multi-hop relaying) to reach as many following vehicles as possible, so they can reschedule or reroute themselves when possible and necessary. One intention of the multi-channel design in IEEE 802.11p DSRC is to achieve this effect by communicating with vehicles both nearby and further away through channel hopping.

Although contention-free MAC schemes, such as time, frequency, or code-division multiple access, have been proposed, the synchronization between nodes in contention-free MAC is hard to achieve and scale, given the ad-hoc nature of VANET and the lack of infrastructures in certain scenarios (e.g., interstate highways across rural areas). In this paper, therefore, we focus on IEEE 802.11p-like contention-based MAC, but operating only in a single channel. For analysis tractability, we simplify the V2V MAC to $p$-persistent CSMA, but the same approach also applies to EDCA or other MAC protocols.

Existing studies show that the network connectivity in one-dimensional spaces (e.g., a highway segment) is always limited, while for two-dimensional spaces (e.g., city blocks), network connectivity can be guaranteed if the density among nearby nodes is above a certain threshold (the so-called percolation phenomenon) [10]. Thus in this paper we focus on a highway segment, where reverse traffic is explored to extend network connectivity and disseminate EMs to vehicles further away. Using reverse traffic to extend cluster size and dealing with its side effect are not entirely new [13], [14]; however, [13] only gave the upper and lower bounds of the average "re-healing" time across disconnected clusters, while we are able to obtain its distribution and calculate the probability of vehicles missing their next detour exit. The work in [14] is to avoid unnecessary message handovers between forward and reverse traffic and can be applied in the TLC framework as well if such scenarios appear.

In [15], nodes were assumed uniformly distributed in $[0, z]$ forming a one-dimensional ad-hoc, multi-hop radio network. By using Laplace Transforms, they derived the probability of network connectivity as a function of transmission range. In our work, however, we assume that the inter-vehicle distance follows a statistical distribution. By their assumption, the number of vehicles within the considered range is a constant; while using our model, this quantity is a random variable. Even with the same vehicle density, their results are more optimistic than ours since the traffic they considered is less bursty. In [16], the connectivity distribution in one-dimensional ad-hoc networks 
was derived, by using an equivalent $G I|D| \infty$ queuing model. The results were obtained by Laplace-Stieltjes Transforms (LST), and simplification was used to get the closed-form expression. In Section IV, we discuss and compare their model with ours. Derivation and simulation show that our approach is simpler, and the results are much more accurate.

Reference [17] proposed an algorithm to dynamically adapt the transmission range of vehicles based on the local density estimation. While this model allows vehicles to estimate local conditions and distinguish between free-flow and congested traffic, our SMC scheme is able to achieve this purpose in the physical layer using only one broadcast, without adjusting the transmission range. In [18], the fraction of vehicles that belong to the largest connected component of a two-dimensional graph was analyzed. Their work, however, did not consider how far the important notification message can reach asymptotically. In [19], we examined the network connectivity in a two-dimensional grid without considering vehicle mobility.

\section{Spatio-Temporal Vehicle Traffic Models}

So far many vehicle mobility models have been proposed, including the car following model and other variants. Through statistical analysis of empirical data collected from real scenarios, the authors of [12] found that an exponential model is a good fit for highway vehicle traffic in terms of inter-vehicle distance and time distribution. Given a single parameter, the vehicle density $\lambda$, this model is able to describe the characteristics and variation of highway traffic, although $\lambda$ is time and location dependent. In this paper, we use the same traffic model and assume the inter-vehicle distance follows an exponential distribution with mean $1 / \lambda$, or equivalently, a Poisson point process with intensity $\lambda$.

For simplicity, we assume a single travel lane in each highway direction; the forward and reverse lanes are parallel to each other and close enough for radio communications. We also assume the reverse traffic has the same density as the forward traffic. It is not difficult to extend the analysis and evaluation to cover the cases of multiple lanes with different vehicle densities. For example, multiple lanes can be treated as a single lane with higher traffic density. When lanes in opposite directions diverge, the reverse traffic can be modeled as a thinning process to reflect its opportunistic nature. Similarly, the model can be extended to handle heterogeneous traffic densities in both directions.

\section{SYSTEM MODEL}

In this section, we first present the TLC framework for EM dissemination, and then show how to use the SMC scheme to help achieve the goal by taking both the time and location criticality into account.

\section{A. TLC-based EM Dissemination}

Figure 1 depicts the scenario for our time/location-critical EM dissemination framework. The $x$-axis shows the distance from the POI at 0 . For example, vehicle $a$ has an accident at time 0 and broadcasts an emergency message with SMC, which allows the message to be delivered over short distance with more details (SDMD) and over long distance with less details (LDLD) at the same time. How SMC achieves this effect will be detailed in the next subsection. Here, we denote the short and long transmission distance as $d_{1}$ and $d_{2}$, respectively. $d_{1}$ has to be greater than a distance thresholdthe reaction deadline $D_{1}$, which in turn depends on the vehicle stopping distance given the human/vehicle reaction time and travel speed. That is, any vehicles within $D_{1}$ from the POI must be notified immediately to avoid the pileup accident. The $y$-axis shows the time deadline at different locations, and the slope of "reaction deadline" is equal to $1 / v_{\max }$, where $v_{\max }$ is the speed limit. To ensure all vehicles receive the SDMD message before the time-location dependent deadline, the POI rebroadcasts the SDMD message periodically every $\tau$ seconds, with $d_{1} \geq D_{1}+\tau v_{\max }$ to accommodate the rebroadcast interval. In this paper, we let $d_{1}>200 \mathrm{~m}$, which is reasonable under the IEEE 802.11p DSRC framework.

On the other hand, the LDLD message should be rebroadcast further to reach as many vehicles as possible. In Fig. 1, vehicle $b$ receives both the SDMD and LDLD messages, and $c$ receives only the LDLD message from the POI. Ideally, $c$ will rebroadcast the LDLD message after a very small delay [20] compared with the POI's rebroadcast interval $\tau$, assuming all vehicles know their location and all messages contain location information. The same process repeats at vehicle $d$. However, when $d$ rebroadcasts, the following vehicle $g$ in the same direction is not close enough (i.e., the inter-vehicle distance is beyond $d_{2}$ ), but $e$ in the opposite direction is in the transmission range of $d$. Therefore, $e$ will rebroadcast the message to reach $f$. The opportunistic use of reverse traffic effectively extends the range of LDLD messages to reach more vehicles in the forward direction, when possible and necessary.

As demonstrated analytically later, even relaying by reverse traffic cannot guarantee to reach a vehicle arbitrarily away from the POI. For example, in Fig. 1, there is no vehicle in either forward or reverse traffic close enough to $f$ to further propagate the LDLD message. Thus the message has to be carried by $f$ itself in the opposite direction and periodically rebroadcast at interval $\tau$. Since the vehicle travel speed is much lower than the speed of electromagnetic waves, the LDLD message incurs a much higher propagation delay, which is dominated by the gap between forward and backward clusters and the rebroadcast interval. Relative to forward traffic, however, the message seems to travel at the doubled vehicle speed, which is shown by the "LDLD progress" staircase curve in Fig. 1. The purpose of the LDLD message dissemination is to reach another distance threshold $D_{2}$, where a detour exit is available, early enough to minimize the probability that vehicles (e.g., g) miss the detour deadline. In Fig. 1, once the LDLD staircase progress curve intersects with the detour deadline curve, any vehicles before the projection of this intersection on the $x$-axis and after $D_{2}$ will miss their detour deadline, since the LDLD message did not reach them in time. In this example, the LDLD message reaches vehicle $h$ before its detour deadline, so $h$ will have the choice to take the detour exit, or travel along the same route and receive the SDMD message with more details later. In this paper, we let $D_{2} \in[1,10] \mathrm{km}$, motivated by the real highway exit distribution [21], for the purpose of performance evaluation. 


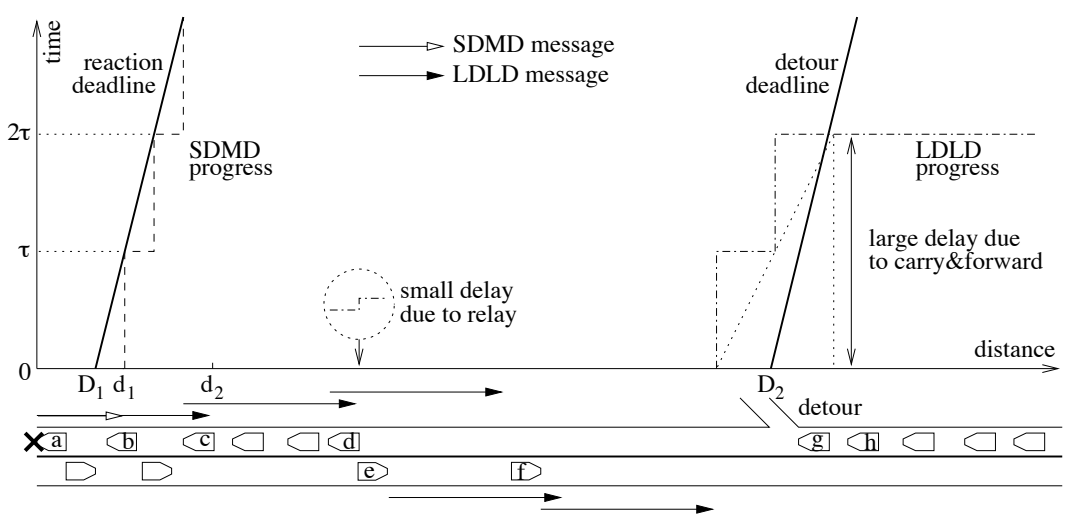

Fig. 1. Time-Location Critical Emergency Message Dissemination Scenarios.

There are other variants of the TLC framework. For example, in addition to LDLD, SDMD messages can also be relayed by both forward and reverse traffic when possible, probably with more intermediate vehicles. Further, more types of messages can be introduced and propagated different distances away from the POI. To keep focused, we only use SDMD/LDLD in this paper to illustrate the TLC framework. In VANET, contention-based MAC protocols need to resolve collisions, which result in random access delay. Since the MAC delay is much smaller than $\tau$ and the travel delay, we can ignore the MAC delay within the same cluster as shown in Fig. 1. Many previous research efforts have addressed how to design effective MAC protocols for reliable EM dissemination and how to quantify their performance [22]. These efforts are orthogonal to our work, and their delay bounds can be incorporated into our analysis as well.

\section{B. Scalable Modulation and Coding}

Traditional physical-layer modulation and coding schemes such as BPSK, QPSK, 16-QAM and 64-QAM map information bits to symbols in the modulation constellation, regardless of the importance of each bit. Although adaptive modulation and coding (AMC) [23] can change modulation and coding schemes depending on the feedback from the receiver, it still treats all information bits equivalently in a particular scheme. However, it is possible to strategically arrange the modulation constellation such that, given the received SINR, the decoded bits of each symbol have different bit error rates (BER). Therefore, some bits can be decoded correctly at longer distances from the transmitter than others. We call this approach scalable modulation and coding (SMC) [9], since it combines different modulation schemes in a scalable way to send different classes of information bits together. SMC is similar to superposition coding (SPC) [24], which simply combines the signals of different modulation schemes together. From an informationtheoretical perspective, both SMC and SPC can achieve higher maximum sum-rate of the broadcast channel than the timesharing schemes (i.e., modulating and transmitting bits of different layers separately). The performance of SPC and SMC is similar. However, SMC can be implemented in software and use the standard QAM modulation and demodulation hardware, while SPC needs special hardware.

Figure 2 illustrates an SMC design using the 64-QAM constellation. Unlike 64-QAM to encode 6 bits of informa-

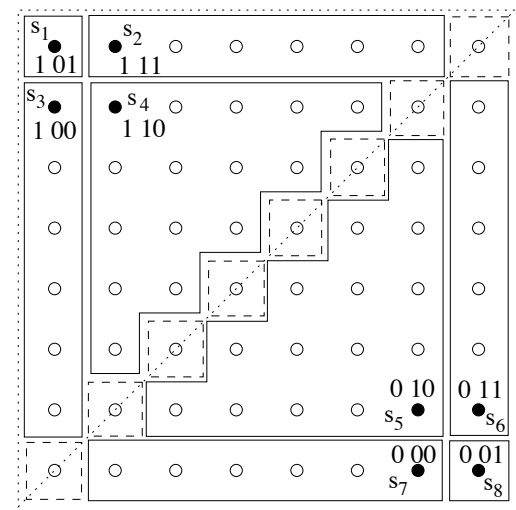

Fig. 2. Scalable Modulation and Coding using the 64-QAM Constellation.

tion without differentiating their importance, here we only encode one bit of information to be transmitted over a longer transmission distance (e.g., the basic information in both SDMD and LDLD, referred to as the layer-1 bit) and two bits of information over a short transmission distance (e.g., the extra information provided in SDMD only, referred to as the layer-2 bits). Although the effective data rate has been halved, the information bits are treated differently in this SMC scheme. The encoder will map these three bits to one of the possible symbols $\left\{s_{1}, s_{2}, \ldots, s_{8}\right\}$ as shown in Fig. 2 . These eight symbols are mapped into two clusters in the constellation (i.e., the cluster encodes the layer-1 bit) and each cluster has four symbols (i.e., they encode the layer-2 bits). The constellation is determined by the intended transmission distance and acceptable BER of layer- 1 and layer- 2 bits. Here we use IEEE $802.11 \mathrm{p}$ as a reference to reach around $200 \mathrm{~m}$ and up to $1 \mathrm{~km}$ for layer-2 and layer-1 bits, respectively.

For the decoder, it will first decode in the same way as 64-QAM, i.e., mapping the received signal to one of the 64 possible symbol locations. In the case shown in Fig. 2, depending on whether the received symbol falls in the upperleft or lower-right triangle, it can decode the layer-1 bit. If the received symbol is along the diagonal line, the layer-1 bit will be decoded arbitrarily. For the layer-2 bits, they are decoded based on which enclosed area they are located in. From the figure, it is clear that layer- 2 bits are more vulnerable to noise and interference, and to achieve the same BER, they should have a higher SINR; or if with the same transmission power, they should be decoded at a shorter distance. This fits 


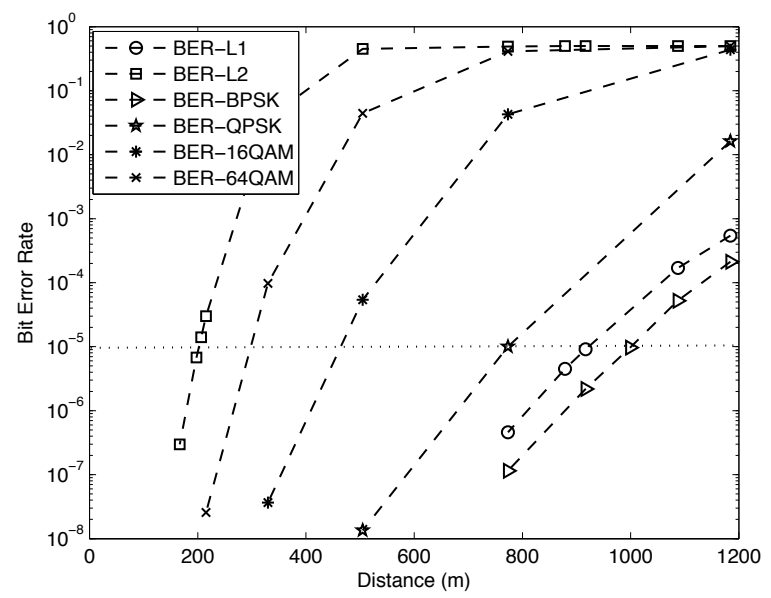

Fig. 3. Bit Error Rate at Different Distances for the SMC Scheme in Fig. 2.

well with the requirement of SDMD messages in the TLC framework.

\section{SMC Performance in the TLC Framework}

Figure 3 shows the achieved BER through simulation for layer-1 (L1) and layer-2 (L2) bits of the SMC scheme depicted in Fig. 2, as well as the BER for ordinary BPSK, QPSK, 16-QAM and 64-QAM with the same transmission power $(33 \mathrm{dBm})$. The path loss model in our simulation follows the ETSI ERM report [25]. Without considering antenna gains, the average received power can be calculated as $P_{e}=P_{s}+L_{0}+L_{l}$, where $P_{e}$ is the received power in $d B m, P_{s}$ is the transmitted power in $d B m$, and $L_{0}$ is the path loss in $d B$ up to the break-point distance $d_{0}$. At $5.9 \mathrm{GHz}$, the break-point distance is given as $d_{0}=15 \mathrm{~m}$ and accordingly $L_{0}=-71 \mathrm{~dB}$. $L_{l}$ is given by $L_{l}=-10 \log \left(d / d_{0}\right)^{n} d B$, where $d$ is the distance between the transmitter and receiver, and $n$ is the path loss exponent (typically 2.7 for vehicular communication environments [26]). In our simulation, the OFDM-based IEEE 802.11p physical layer is used: one FFT frame contains 52 data subcarriers and 12 guard and pilot subcarriers with central frequency at $5.9 \mathrm{GHz}$. Rayleigh fading is simulated to reflect the channel fluctuation due to fast fading. Besides the SMC scheme, the transceiver also adopts a $1 / 2$ convolution code and random interleaver for error correction purposes.

From the simulation, $8.5 d B$ and $25.5 d B$ are the SINR threshold needed for L1 and L2 bits to achieve the $10^{-5}$ BER requirement, respectively. This SINR translates into an $879 \mathrm{~m}$ and $206 \mathrm{~m}$ transmission distance for L1 and L2 bits, respectively, which meets the requirement of IEEE 802.11p DSRC. More importantly, it is achieved by a single transmission to send one L1 bit and two L2 bits to different distances at the same time. We could do so with QPSK in the first half slot to send one L1 bit and with 16-QAM in the second half slot to send two L2 bits. With a contentionbased MAC, more transmission attempts will increase channel contention and collision, thus increasing media access delay. Also as shown in Fig. 3, L1 reaches further than QPSK at the cost of a shorter distance of L2 than 16-QAM, without changing modulation schemes. The tradeoff is well justified in the following section when considering SDMD and LDLD connectivity. For an optimization framework to design SMC schemes and make such tradeoff, please refer to [9].

\section{Performance Analysis and Evaluation}

In this paper, a cluster is a "connected" group of vehicles on a one-dimensional highway, in which either SDMD or LDLD can be relayed by radio transmissions. The distance between the first and the last vehicles in the same cluster, or cluster size, is of great importance in SDMD and LDLD dissemination for collision avoidance and look-ahead decision making. In this section, we first derive the cluster size and distribution, then show how to use reverse traffic to extend the cluster size, and finally examine the properties of LDLD/SDMD messages in the TLC framework using the SMC scheme.

\section{A. Cluster Size Characterization}

1) Moments of Cluster Size: Reference [16] analyzed the connectivity in one-dimensional ad-hoc networks using an equivalent $G I|D| \infty$ queuing model, where node positions are equivalent to customer arrivals, and the transmission range is modeled as a constant service time. Therefore, with an infinite number of servers, connected components in a onedimensional network are actually the "busy period" of a $G I|D| \infty$ queue. The authors of [16] obtained the expected cluster size by means of Laplace-Stieltjes Transforms (LST) and some simplification, which are in turn based on the derivation in [11]: $E[B]=R+\frac{\int_{0}^{R} x f_{X}(x) \mathrm{d} x}{1-F_{X}(R)}$, where $E[B]$ is the expected cluster size, $R$ is the transmission range, and $F_{X}(x)=\int_{-\infty}^{x} f_{X}(x) \mathrm{d} x$ is the cumulative distribution function (CDF) of the inter-node distance. Assuming a Poisson point process, the exponential inter-arrival distribution with mean $1 / \lambda$ gives

$$
E[B]=R+\frac{\int_{0}^{R} \lambda x e^{-\lambda x} \mathrm{~d} x}{e^{-\lambda R}}=\frac{1-e^{-\lambda R}}{\lambda e^{-\lambda R}} .
$$

Different from the above approximated result, we have a simple approach, which obtains the exact average cluster size without any simplification or approximation. By only using the i.i.d. property of the inter-vehicle distance confirmed in [12], we will also show that our approach is much more accurate. Denote $C$ as the random variable for the cluster size, we have the following recursion to obtain the expectation of $C$ :

$$
\begin{array}{r}
E[C]=\operatorname{Pr}\left\{X_{1}<R\right\} \times\left(E\left[X_{1} \mid X_{1}<R\right]+E[C]\right) \\
+\operatorname{Pr}\left\{X_{1} \geq R\right\} \times 0,
\end{array}
$$

where $X_{1}$ is the distance between the first and second vehicles in the same cluster (i.e., the definition of $E[C]$ is recursive), and $\operatorname{Pr}\left\{X_{1}<R\right\}=1-e^{-\lambda R}$. Let ${\overline{X^{\prime}}}_{1}=E\left[X_{1} \mid X_{1}<R\right]=$ $\int_{0}^{R} \lambda x e^{-\lambda x} /\left(1-e^{-\lambda R}\right) \mathrm{d} x$, we have

$$
E[C]=\frac{1-e^{-\lambda R}}{e^{-\lambda R}} \times \overline{X^{\prime}}{ }_{1}=\frac{1-e^{-\lambda R}(\lambda R+1)}{\lambda e^{-\lambda R}} .
$$

Figure 4 compares the results from (1), (3) and the simulation in Matlab, given the traffic density and transmission range. In Fig. 4, $\lambda$ is 0.01 (number of vehicles per meter), and the $y$-axis is the expected cluster size in log scale. When $\lambda R$ is sufficiently large, both approaches match the simulation 


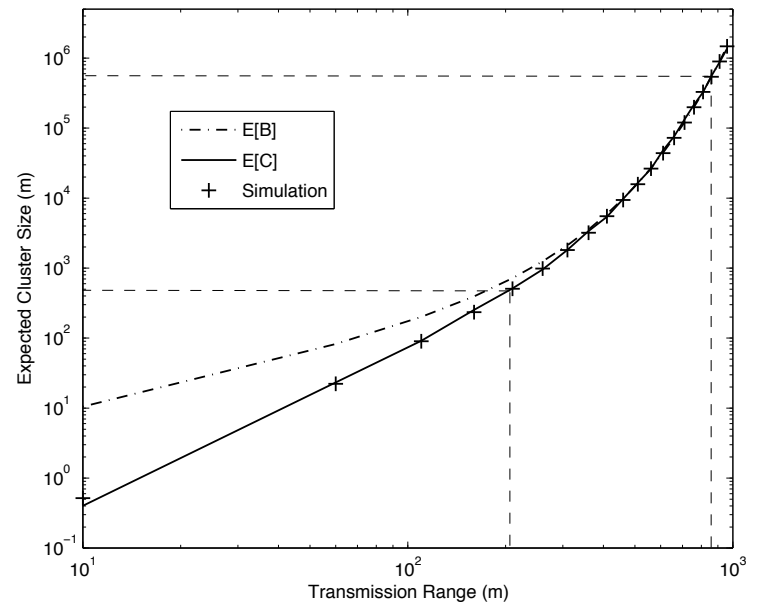

Fig. 4. Comparison between $E[B]$ in [16] and $E[C]$ in Our Approach on the Expected Cluster Size.

well; but for small $R$ and $\lambda$, our approach gives much more accurate results. For example, at $R=206 \mathrm{~m}, E[B]=685 \mathrm{~m}$ and $E[C]=479 \mathrm{~m}$, while the simulation gives $478 \mathrm{~m}$. Reference [13] also obtained the same average cluster size (or cluster length in their terminology) as ours, but our approach is simpler and more direct.

Similarly, we can obtain higher-order moments of $C$. For instance, the second-order moment of $C$ is

$$
\begin{array}{r}
E\left[C^{2}\right]=\operatorname{Pr}\left\{X_{1}<R\right\} \times E\left[\left(C+X_{1}\right)^{2} \mid X_{1}<R\right] \\
=\frac{1-e^{-\lambda R}}{e^{-\lambda R}} \times\left(2 E[C] \overline{X^{\prime}}{ }_{1}+{\overline{X^{\prime}}}_{1}^{2}\right),
\end{array}
$$

where ${\overline{X^{\prime}}}_{1}^{2}=E\left[X_{1}^{2} \mid X_{1}<R\right]=\int_{0}^{R} \lambda x^{2} e^{-\lambda x} \mathrm{~d} x$. Higherorder moments, however, are not discussed in [13].

2) Cluster Size Distribution: Let $X_{i}$ 's be the random variables of the inter-vehicle distance between the $i$-th and the $(i+1)$-th vehicles, which are i.i.d. with $f_{X_{i}}(x)=\lambda e^{-\lambda x}$ for $x>0 . F_{X_{i}}(R)=\int_{0}^{R} f_{X_{i}}(x) \mathrm{d} x=1-e^{-\lambda R}$ is the probability that the consecutive vehicles are within the transmission range of each other. Let $X_{i}^{\prime}$ 's be the random variables of the intervehicle distance, given that the $i$-th and $(i+1)$-th vehicles are in the same cluster, then

$$
f_{X_{i}^{\prime}}(x)=f_{X_{i} \mid 0 \leq X_{i} \leq R}(x \mid 0 \leq x \leq R)=\frac{\lambda e^{-\lambda x}}{1-e^{-\lambda R}}
$$

for $0 \leq x \leq R$. Suppose that there are $k$ vehicles in a cluster, the Laplace Transform of the cluster size distribution is

$$
\begin{array}{r}
f_{C \mid k}^{*}(s)=f_{X_{1}^{\prime}+X_{2}^{\prime}+\ldots X_{k}^{\prime}}^{*}(s) \\
=\left[\frac{\lambda}{1-e^{-\lambda R}} \times \frac{1-e^{-(s+\lambda) R}}{s+\lambda}\right]^{k} .
\end{array}
$$

If $f_{C \mid k}$ can be obtained by taking the inverse-Laplace Transform on (6), then the distribution function of cluster size $C$ is

$$
f_{C}(x)=\sum_{k=1}^{\infty} f_{C \mid k} \operatorname{Pr}\{k\}
$$

where $\operatorname{Pr}\{k\}=\left(1-e^{-\lambda R}\right)^{k-1} e^{-\lambda R}$ is the probability that there are $k$ vehicles in a cluster.

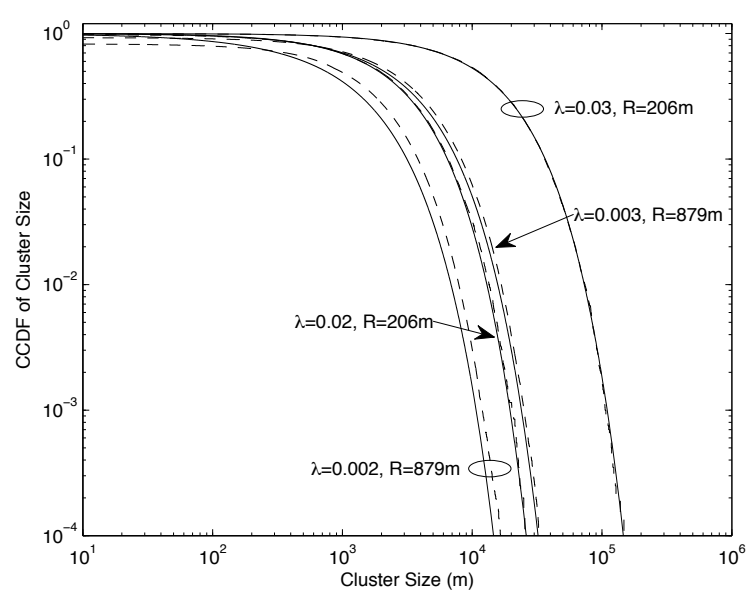

Fig. 5. Gamma Approximation of the Cluster Size Distribution (Solid Curve: Analysis, Dashed Curve: Simulation).

Unfortunately, (6) does not yield any closed-form results by inverse-Laplace Transform. Thus it is very difficult to give the exact formula of the distribution function. In fact, $C$ is the sum of $k$ truncated exponential random variables (given that they are smaller than the threshold $R$ ), and $k$ itself follows a Geometric distribution, which further complicates the derivation.

Although similar to [16], the closed-form distribution of the cluster size cannot be derived, we can obtain its statistical moments without any simplification. Using these moments, we can use some known distributions to approximate the cluster size distribution. We find Gamma distribution is sufficient for this purpose. Similar to the average cluster size in Fig. 4, the cluster size distribution, or the complementary cumulative distribution function (CCDF) in our case, can be obtained according to the first and second-order cluster size moments with different values of $R$ and $\lambda$. In Fig. 5, the dotted lines are from simulation results and the solid lines are from the corresponding Gamma approximation,

$$
f_{C}(x)=x^{k-1} \frac{e^{-x / \theta}}{\theta^{k} \Gamma(k)}, \text { for } x>0,
$$

where $k=\left(E\left[C^{2}\right] / E[C]^{2}-1\right)^{-1}$ and $\theta=E[C] / k$ ensure that the first and second-order moments of the Gamma approximated random variables are the same as $E[C]$ and $E\left[C^{2}\right]$. Both the $x$ and $y$-axis in Fig. 5 are in log scale. Gamma distribution clearly is a good fit for the cluster size distribution, according to the simulation results shown in the figure.

\section{B. Using Reverse Traffic}

By observing the cluster size distribution, we find that there is a non-negligible probability that the size of a cluster is much smaller than its expected value. Thus, the cluster is likely to be so small that a large number of following vehicles are unable to receive the LDLD message. When the propagation of LDLD reaches the end of a cluster, it cannot be forwarded any further unless some vehicles in the opposite direction pick up the message, and carry it towards the vehicles that are far behind. Although this incurs a large delay when compared with that inside a cluster (due to the much lower vehicle speed than 


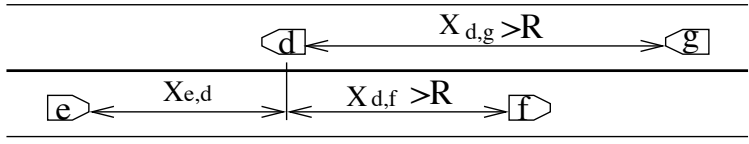

(a) Case 1

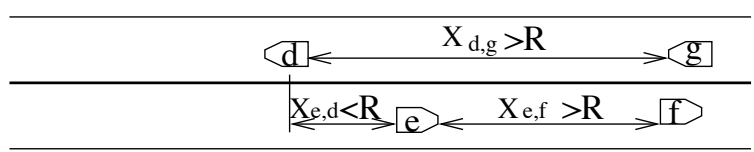

(b) Case 2

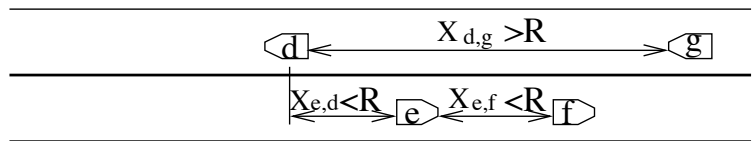

(c) Case 3

vehicle in reverse traffic

Fig. 6. Three Cases to Extend the Forward Cluster by Reverse Traffic.

electromagnetic waves), the reverse traffic can indeed greatly extend the reach of LDLD messages, e.g., [13], [14]. [13] gives the upper and lower bounds of the average "re-healing" time to cross clusters, but in this paper we try to obtain the "carryand-forward" delay distribution. The vehicles in the reverse direction can also form clusters to relay the message quickly, which further complicates the analysis.

Denote $X_{i, j}$ (or $X_{i, 0}$ ) the distance between vehicle $i$ and $j$ (or $i$ and the POI) at time 0 . Without loss of generality, let vehicle $d$ be the last vehicle in a cluster in the forward direction, $g$ be the first vehicle of the next cluster in the same direction, and $e$ be a vehicle in the reverse direction that can help relay or deliver the message to $g$. We consider the following three different cases to derive the distribution of the traveling delay, which dominates the "carry-and-forward" delay using reverse traffic.

1) Case 1: As shown in Fig. 6(a), there is no vehicle located between $d$ and $g$ in the reverse direction and within the transmission range of $d$ at the same time (i.e., $X_{d, f}>R$ ). The message needs to be relayed by $e$ in the reverse direction, and $X_{e, 0}<X_{d, 0}$. The probability for this case to happen is

$$
P_{1}=\operatorname{Pr}\left\{X_{d, f}>R\right\}=e^{-\lambda R} .
$$

In this case, the message must be propagated from $e$ to $g$ when $e$ travels close to $g$. The distance between $e$ and $d$, $X_{e, d}$, follows the exponential distribution, and the distance distribution between $d$ and $g$ is $f_{X_{d, g}}(x)=\frac{\lambda e^{-\lambda x}}{e^{-\lambda R}}$ for $x \geq R$. Let $Y_{1}$ be this travel distance, then $Y_{1}=X_{e, d}+X_{d, g}$ has the distribution

$$
\begin{aligned}
f_{Y_{1}}(y) & =\int f_{X_{e, d}}(x) f_{X_{d, g}}(y-x) \mathrm{d} x \\
& =\lambda^{2}(y-R) e^{-\lambda(y-R)},
\end{aligned}
$$

for $y \geq R$.

2) Case 2: As shown in Fig. 6(b), $e$ is within the communication range of the forward cluster, and it is closer to $g$ than $d$ is. $e$ 's neighbor vehicle $f$ is outside $e$ and $d$ 's transmission ranges, so $f$ cannot relay the message to $g$. Thus, $X_{e, 0} \geq X_{d, 0}$,
$X_{e, d} \leq R$, and $X_{e, f} \geq R$. The probability of this case is

$$
\begin{array}{r}
P_{2}=\operatorname{Pr}\left\{X_{e, d} \leq R\right\} \operatorname{Pr}\left\{X_{e, f} \geq R\right\} \\
=\left(1-e^{-\lambda R}\right) e^{-\lambda R} .
\end{array}
$$

Here, the gap $Y_{2}=X_{d, g}-X_{e, d}$. Since $f_{X_{e, d}}(x)=\frac{\lambda e^{-\lambda x}}{1-e^{-\lambda R}}$ for $0 \leq x \leq R$, and $f_{X_{d, g}}(x)=\frac{\lambda e^{-\lambda x}}{e^{-\lambda R}}$ for $x \geq R$, the distribution of $Y_{2}$ is

$$
\begin{aligned}
f_{Y_{2}}(y) & =\int f_{X_{e, d}}(x) f_{X_{d, g}}(x+y) \mathrm{d} x \\
& =\frac{\lambda}{2}\left\{\begin{array}{lr}
\frac{\left(e^{\lambda y}-e^{-\lambda y}\right)}{\left(e^{\lambda R}-1\right)} & 0 \leq y \leq R, \\
\left(e^{\lambda R}+1\right) e^{-\lambda y} & y \geq R .
\end{array}\right.
\end{aligned}
$$

3) Case 3: As shown in Fig. 6(c), both $d$ and $f$ are within the transmission range of $e$. Thus $f$ (and possibly other vehicles in the same cluster as $e$ and $f$ in the reverse direction) can relay the message further, in order to reach $g$ faster. In this case, $X_{e, 0} \geq X_{d, 0}, X_{e, d} \leq R$, and $X_{e, f} \leq R$. Therefore,

$$
P_{3}=\operatorname{Pr}\left\{X_{e, d} \leq R\right\} \operatorname{Pr}\left\{X_{e, f} \leq R\right\}=\left(1-e^{-\lambda R}\right)^{2} .
$$

Let $Y_{3}=\max \left\{0, X_{e, g}-C^{\prime}\right\}$, where $C^{\prime}$ is how far the message can be relayed within the cluster in the reverse direction, which has the same distribution as cluster size $C$. The density function of $X_{e, g}$ is derived in Case 2 as $f_{Y_{2}}(y)$, so $f_{Y_{3}}(y)$ can be obtained by $f_{Y_{3}}(y)=\int f_{X_{e, g}}(x) f_{C^{\prime}}(x-y) \mathrm{d} x$. To simplify the calculation, we assume $C^{\prime}$ is a constant with value $E[C]$. Let $\delta$ be the Dirac delta function, thus the distribution of $Y_{3}$ is approximated by

$$
f_{Y_{3}}(y) \approx \begin{cases}f_{Y_{2}}(y+E[C]) & y \geq 0, \\ \delta \int_{0}^{E[C]} f_{Y_{2}}(x) \mathrm{d} x & y=0 .\end{cases}
$$

Based on the above three cases, the density function of the travel distance is $f_{Y}(y)=\sum_{i=1}^{3} P_{i} \times f_{Y_{i}}(y)$. Since messages can be directly transmitted between two vehicles that are within transmission range of each other, the actual distance that will lead to the message propagation delay is $\max \{0, Y-R\}$, and the delay $D$ for the reverse traffic to deliver the message to the next forward cluster has the CDF

$$
F_{D}(x)=F_{Y}(2 x v+R) \quad \text { for } x \geq 0,
$$

where $v$ is the speed of the vehicles in both directions.

Figure 7(a) shows the comparison between our analysis and the simulation results by $\operatorname{CCDF} \overline{F_{D}}(x)=1-F_{D}(x)$. In the simulation, traveling delay is calculated according to the distance between clusters and the given vehicle speed, $120 \mathrm{~km} / \mathrm{h}$ in our case. Traffic density $\lambda$ is measured by the number of vehicles per meter. Although we simplify the cluster size by its expectation $E[C]$ obtained in (3), the analysis and simulation match quite well. Compared with the results in [13] on the upper and lower bound of the average "re-healing" time in Fig. 7(b), our work obtaining the "carry-and-forward" delay distribution is a considerable further effort. Our results, shown by the Analysis curve, are more accurate at low traffic density. Note that this delay, in the order of seconds, is much larger than the message propagation and MAC access delay within a cluster, which will be evaluated in Section IV-E. 


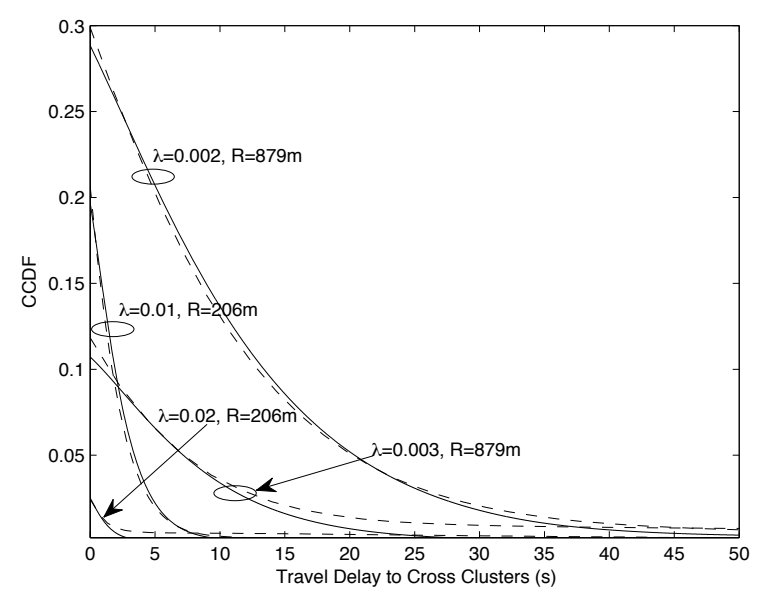

(a) Travel Delay due to Reverse Traffic to Cross Forward Clusters (Solid Curve: Analysis, Dashed Curve: Simulation).

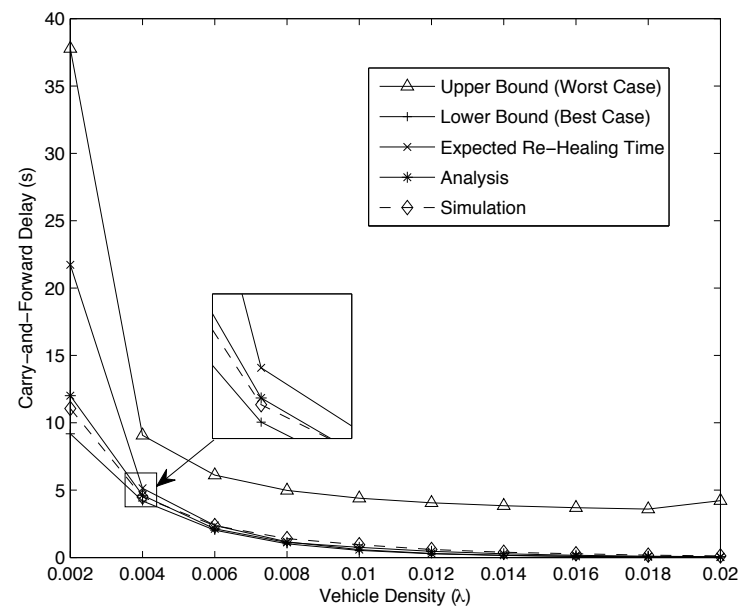

(b) Travel Delay Comparison with [13], $R=206 \mathrm{~m}$

Fig. 7. Travel Delay to Cross Forward Clusters and Comparison with [13].

\section{Probability to Miss LDLD Deadlines}

We assume that the location of highway exits, i.e., $D_{2}$ in Fig. 1, is known by using either digital map or GPS. For a tagged vehicle $g$ in Fig. 1, which is $X_{g, D_{2}}$ away from the exit $D_{2}$ at time 0 , the deadline to receive the LDLD message before $g$ passes by the detour exit is $t_{D}=\left(X_{g, D_{2}}\right) / v$. Let $C_{1}$ be the size of the cluster containing the POI, and $C_{k}$ be the size of the $k$-th cluster in the forward direction. $C_{k}$ 's are i.i.d. random variables with the distribution given in (7). Let $G_{k}$ be the distance gap between $C_{k}$ and $C_{k+1}$, the distribution of $G_{k}$ is $f_{G_{k}}(x)=\lambda e^{-\lambda(x-R)}$ for $x \geq R$.

The probability that $g$ is in the first cluster is $1-F_{C}\left(X_{g, 0}\right)$. For $k>1, g$ is in the $k$-th cluster when $X_{g, 0}$ is larger than the sum of cluster sizes plus inter-cluster gaps $\sum_{i=1}^{k-1}\left(C_{i}+G_{i}\right)$ but smaller than $\sum_{i=1}^{k-1}\left(C_{i}+G_{i}\right)+C_{k}$. Thus the probability that $g$ is in $C_{k}, \operatorname{Pr}\left\{g \in C_{k}\right\}$, is

$$
\int_{0}^{X_{g, 0}} f_{\sum_{i=1}^{k-1}\left(C_{i}+G_{i}\right)}(x)\left[1-F_{C_{k}}\left(X_{g, 0}-x\right)\right] \mathrm{d} x .
$$

The delay for the reverse traffic to carry the message from $C_{k}$ to $C_{k}+1$ is $D_{k}$, which has the i.i.d. distribution given in (15). The probability that $g$ misses the LDLD message before

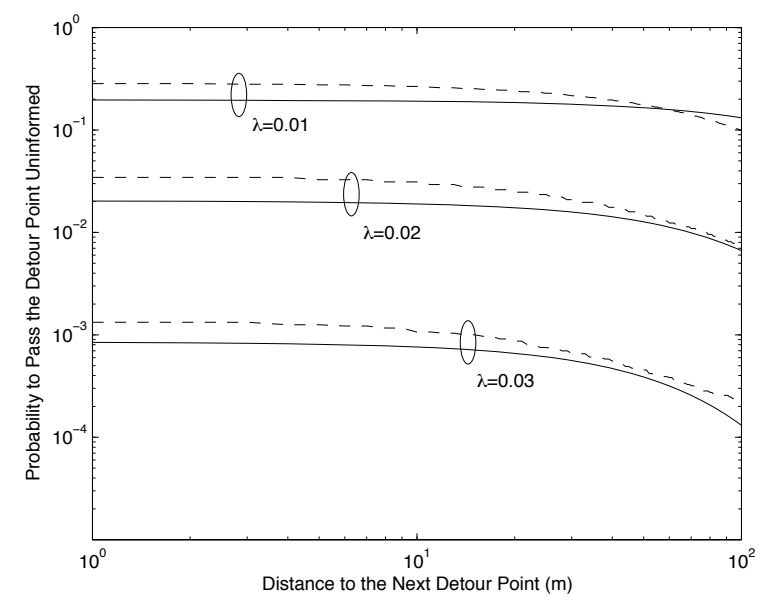

(a) $R=206 \mathrm{~m}, D_{2}=5 \mathrm{~km}$

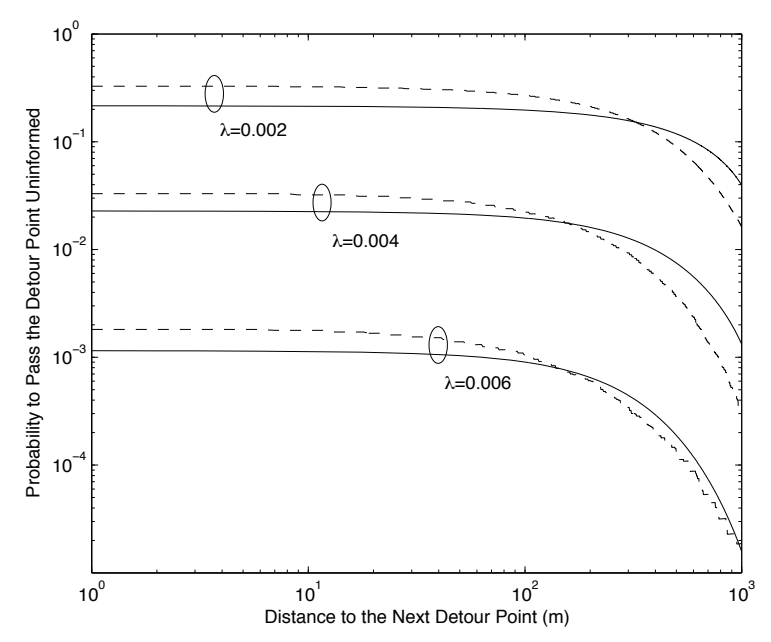

(b) $R=879 \mathrm{~m}, \mathrm{D}_{2}=5 \mathrm{~km}$

Fig. 8. The Probability to Miss LDLD Deadlines (Solid Curve: Analysis, Dashed Curve: Simulation).

its deadline, $\operatorname{Pr}\{g$ miss $L D L D\}$, is thus

$$
\sum_{k=2}^{\infty} \operatorname{Pr}\left\{g \in C_{k}\right\}\left[1-F_{\sum_{i=1}^{k-1} D_{i}}\left(t_{D}\right)\right] .
$$

When $k$ is large enough, we can use Gaussian random variables to approximate $\sum_{i=1}^{k-1}\left(C_{i}+G_{i}\right)$ and $\sum_{i=1}^{k-1} D_{i}$ in order to simplify the calculation.

Figure 8(a) and (b) show the analytical and simulation results for the probability to miss LDLD deadlines, versus the distance between a vehicle and a highway exit. In both figures, the location of the exit is $5 \mathrm{~km}$ from the POI. When traffic density $\lambda$ is higher, it is more likely that the reverse traffic can help disseminate LDLD messages and extend the cluster size in the forward direction, therefore the probability to miss LDLD deadline is much lower (note that the $y$-axis is in log scale and the gap in the lower portion of the figure is actually smaller). Radio transmission range, on the other hand, also affects the cluster size. Thus using a larger $R$ to cover longer distances can also reduce the risk of a vehicle missing LDLD messages before passing by an exit, which substantiates our choice of using the SMC scheme. The simulation results match our analysis well. 


\section{Guaranteeing SDMD Deadlines}

It is important and relatively simpler to guarantee the delivery of SDMD messages within the deadline to avoid collision. Typically, the stopping distance of a vehicle is determined by human perception and reaction time $(2 \sim 4$ seconds), vehicle reaction time and vehicle brake distance. Using SDMD messages and automatic collision avoidance, we can save the human perception and reaction time, and consider the vehicle reaction time and brake distance only for the stopping distance $D_{1}$. As discussed earlier, to guarantee the SDMD messages to be received by others before they are within the stopping distance of the POI, the transmission range of the SDMD message must be greater than $D_{1}+\tau v$. Using the highway traffic speed of $120 \mathrm{~km} / \mathrm{h}(\approx 33.33 \mathrm{~m} / \mathrm{s})$ as an example, the vehicle reaction and braking distance is less than $100 \mathrm{~m}$. Using the SMC scheme proposed in Section III-B, the transmission distance of L2 bits can reach more than $206 \mathrm{~m}$. Therefore, $206 m>100 m+\tau \times 33.33 \mathrm{~m} / \mathrm{s}$. Thus we set $\tau<3.18$ seconds (minus the maximum MAC layer delay) to ensure that the SDMD message can be delivered to all of the following vehicles before their reaction deadline.

\section{E. Intra-Cluster Behaviors}

Ideally, emergency messages will be propagated inside a cluster from the beginning to the end without any delay other than electromagnetic wave propagation. However, MAC protocols need to coordinate the transmission among nodes to avoid packet collision when accessing the wireless medium. For tractable analysis, we have simplified the MAC protocol to a $p$-persistent CSMA protocol, in which each active node broadcasts with probability $p$ at the beginning of each time slot when the channel is sensed idle. Each idle time slot has a constant duration (e.g., $13 \mu \mathrm{s}$ in IEEE 802.11p), and each busy time slot corresponds to the transmission time of an SDMD or LDLD message (less than $1 \mathrm{~ms}$ for an emergency message of 100 bytes). The maximum advancement of a message in a single time slot is the transmission range $R$, and the message will be propagated until it reaches the end of a cluster.

For broadcast messages, we cannot rely on link-layer acknowledgment messages to identify whether a transmission is successful or not. Here, each node that receives an emergency message will attempt to relay (rebroadcast) the message until it overhears the same message being transmitted by a node that is further away from the POI, using the information from onboard GPS receivers and digital maps. If a node has retransmitted a message up to the MAC layer retry limit and still cannot hear the same message being broadcast by others further away (probably due to the fact that it is already the last vehicle in a cluster), it will wait for a time interval $\tau$ and retry again. Now we examine the delay within a cluster using the above simplified MAC protocol and relay scheme.

Figure 9(a) shows the case with $\lambda=0.01, R=206 \mathrm{~m}$ and $p=1 /(\lambda R)$. The larger the number of time slots (TS), the further the message propagates across the cluster, but it is bounded by the cluster size (the asymptotic bound in the figure). E.g., at time slot 32, the propagation distance is quite close to the bound. Figure 9(b) is the case where the farthest node in each transmission always has probability 1

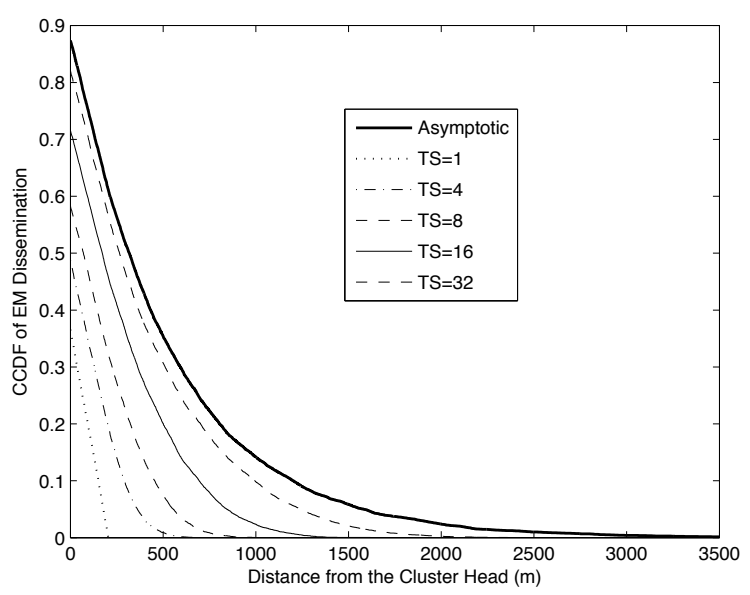

(a) With the p-persistent CSMA.

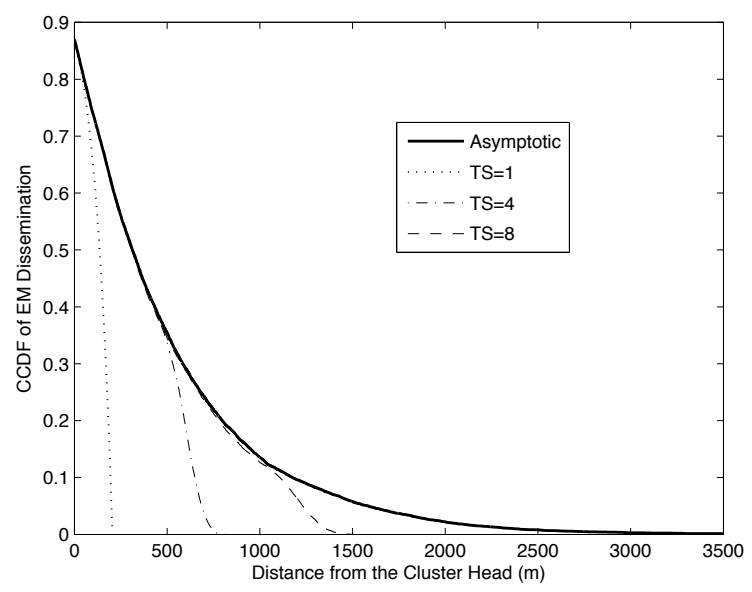

(b) With the furthest-first MAC.

Fig. 9. Emergency Message Dissemination Progress inside a Cluster.

to rebroadcast. As the best possible case, it needs fewer time slots to get close to the asymptotic bound.

If we do a worst-case intra-cluster delay estimation, the idle slot duration equals $13 \mu \mathrm{s}$ and the busy slot duration is $1 \mathrm{~ms}$. If 64 time slots are needed to propagate the message to the end of a cluster, then the worst-case delay inside a cluster will be $48 \mathrm{~ms}$. This is negligible when compared with the travel delay in Fig. 7 due to the "carry-and-forward" propagation when crossing clusters, which is typically in tens of seconds. This confirms that when we analyze the probability of furtheraway vehicles missing their detour deadlines, we can safely ignore the delay within each cluster.

\section{Conclunsions}

In this paper, we proposed a time/location-critical (TLC) framework for emergency message (EM) dissemination in vehicular ad-hoc networks (VANET). Although data dissemination in VANET and MANET has been studied extensively in the literature, to the best of our knowledge, it is the first time that multiple deadlines at different locations are taken into account at the same time. This is achieved through our previously proposed SMC scheme, which allows messages of different importance to be broadcast to different distances simultaneously. This unique feature fits well with 
the requirement of instant collision avoidance and advanced travel planning in VANET. More importantly, we obtained the exact average cluster size using a simpler approach, as well as a close approximation to its distribution. We also analyzed more rigorously the benefit of using reverse traffic to extend the cluster size in terms of the delay distribution. Extensive simulation results have shown the efficacy of the TLC framework and its performance analysis. Our future work will focus on optimizing the SMC parameters to meet a wider range of VANET applications, as well as extending the existing TLC framework from one-dimensional highway to a two-dimensional scenario, which is a more challenging problem.

\section{ACKNOWLEDGMENT}

This work is supported in part by the Natural Sciences and Engineering Research Council of Canada (NSERC), Canada Foundation for Innovation (CFI) and British Columbia Knowledge Development Fund (BCKDF).

\section{REFERENCES}

[1] "Dedicated Short Range Communications (DSRC) Home," [Online]. http://www.leearmstrong.com/DSRC/DSRCHomeset.htm

[2] J. Blum, A. Eskandarian, and L. Hoffman, "Challenges of inter-vehicle ad hoc networks," IEEE Trans. Intell.Transportation Syst., 5(4):347-351, 2004

[3] T. Nadeem, P. Shankar, and L. Iftode, "A comparative study of data dissemination models for VANETs," in ICST MobiQuitous'06, 2006.

[4] S. Biswas, R. Tatchikou, and F. Dion, "Vehicle-to-vehicle wireless communication protocols for enhancing highway traffic safety," IEEE Commun. Mag., vol. 44, no. 1, pp. 74-82, Jan. 2006.

[5] F. Li and Y. Wang, "Routing in vehicular ad hoc networks: A survey," IEEE Veh. Technol. Mag., 2(2):12-22, 2007.

[6] G. Resta, P. Santi, and J. Simon, "Analysis of multi-hop emergency message propagation in vehicular ad hoc networks," in Proc ACM MobiHoc'07, Montreal, Quebec, Canada, 2007.

[7] C. Chiasserini, et. al., "Smart broadcast of warning messages in vehicular ad hoc networks," in Proc. Workshop Interno Progetto NEWCOM (NoE'05), 2005.

[8] D. Jiang and L. Delgrossi, "IEEE 802.11p: Towards an international standard for wireless access in vehicular environments," in IEEE VTC'08 Spring, 2008.

[9] L. Cai, Y. Luo, S. Xiang, and J. Pan, "Scalable modulation for scalable wireless videocast," IEEE INFOCOM-Mini'10, 2010.

[10] O. Dousse, "Asymptotic properties of wireless multi-hop networks," Ph.D. Thesis, EPFL, 2005.

[11] L. Liu and D.-H. Shi, "Busy period in $G I^{X}|G| \infty$," J. Appl. Prob., vol. 33, pp. 815-829, 1996.

[12] F. Bai and B. Krishnamachari, "Spatio-temporal variations of vehicle traffic in VANETs: facts and implications," in Proc. ACM International Workshop on VehiculAr InterNETworking (VANET'09), 2009.

[13] N. Wisitpongphan, F. Bai, P. Mudalige, V. Sadekar, O. Tonguz, "Routing in sparse vehicular ad hoc wireless networks," IEEE J. Sel. Areas Commun., vol. 25, no. 8, Oct. 2007.

[14] M. Abuelela and S. Olariu, "SODA: a smart opportunistic data dissemination approach for VANET," in Proc. of the International Workshop on Intelligent Transportation, 2009.

[15] M. Desai and D. Manjunath, "On the connectivity in finite ad hoc networks," IEEE Commun. Lett., 6(10):437-439, 2002.

[16] D. Miorandi and E. Altman, "Connectivity in one dimensional ad hoc networks: a queuing theoretic approach," ACM/Springer Wireless Networks, 2006.

[17] M.M. Artimy, W.J. Phillips, and W. Robertson, "Assignment of dynamic transmission range based on estimation of vehicle density," in Proc. ACM Int'l Workshop on VehiculAr InterNETworking (VANET'05), 2005.

[18] M. Kafsi, P. Papadimitratos, O. Dousse, T. Alpcan, and J.-P. Hubaux, "VANET Connectivity Analysis," in Workshop on Automotive Networking and Applications (Autonet), 2008.

[19] Y. Zhuang, J. Pan, and L. Cai, "A probabilistic model for message propagation in two-dimensional vehicular ad-hoc networks," in Proc. ACM VANET'10, pp. 31-40, 2010.
[20] G. Korkmaz, E. Ekici, F. Ozguner, and U. Ozguner, "Urban multihop broadcast protocol for inter-vehicle communication systems," in Proc. ACM International Workshop on VehiculAr InterNETworking (VANET'04), pp. 76-85, 2004.

[21] "List of exits on Highway 401 (Ontario)," [Online]. http://en.wikipedia. org/wiki/List_of_exits_on_Highway_401_(Ontario)

[22] Y. Bi, H. Zhao, and X. Shen, "A Directional Broadcast Protocol for Emergency Message Exchange in Inter-Vehicle Communications," in Proc IEEE ICC'09, Dresden, Germany, June 2009.

[23] K.-B. Song, A. Ekbal, S.T. Chung and J.M. Cioffi, "Adaptive modulation and coding (AMC) for bit-interleaved coded OFDM (BIC-OFDM)," in Proc IEEE ICC'04, 2004.

[24] S. Bopping and J.M. Shea, "Superposition coding in the downlink of CDMA cellular systems," in Proc IEEE WCNC'06, 2006.

[25] ETSI ERM Committee. "Technical Characteristics for Pan-European Harmonized Communication Equipment Operating in the $5 \mathrm{GHz}$ Frequency Range and Intended for Critical Road-Safety Applications," Technical Report ETSI TR 102 492-1/2, ETSI, 2005.

[26] L. Le, W. Zhang, A. Festag, and R. Baldessari, "Analysis of approaches for channel allocation in car-to-car communication," in Proc 1st International Workshop on Interoperable Vehicles (IOV'08), pp. 33-38, 2008.

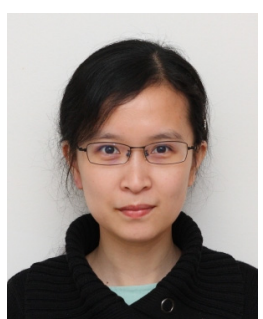

Yanyan Zhuang (S'09) received her Bachelor's and M.E. degrees in Computer Science from Southeast University, Nanjing, China, in 2005 and 2008, respectively. She is currently a Ph.D. candidate at the Department of Computer Science, University of Victoria, British Columbia, Canada. Her research interests span the areas of network protocols, system design and analysis in wireless communications and technologies, especially in Wireless Sensor Networks and Vehicular Ad-Hoc Networks.

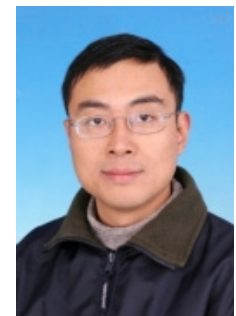

Jianping Pan (S'96-M'98-SM'08) is currently an assistant professor of computer science at the University of Victoria, British Columbia, Canada. He received his Bachelor's and $\mathrm{PhD}$ degrees in computer science from Southeast University, Nanjing, China, and he did his postdoctoral research at the University of Waterloo, Ontario, Canada. He also worked at Fujitsu Labs and NTT Labs. His area of specialization is computer networks and distributed systems, and his current research interests include protocols for advanced networking, performance analysis of networked systems, and applied network security. He is a senior member of the ACM.

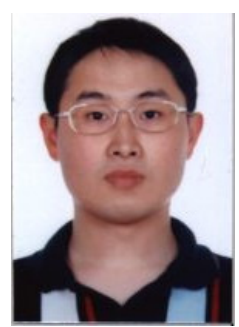

Yuanqian Luo (S'09) received his B.S. and M.S. degrees in Electrical Engineering from Southeast University, Nanjing, China, in 2005 and 2008, respectively. He is currently a Ph.D. student at the Department of Electrical and Computer Engineering, University of Victoria, British Columbia, Canada. His current research interests include cross-layer design and optimization for cooperative networks.

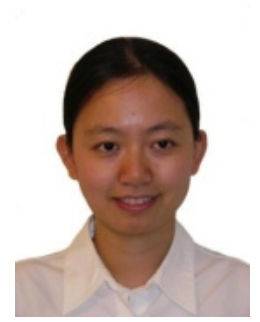

Lin Cai (S'00-M'06) received the M.A.Sc. and $\mathrm{Ph} . \mathrm{D}$. degrees (with Outstanding Achievement in Graduate Studies Award) in electrical and computer engineering from the University of Waterloo, Waterloo, Canada, in 2002 and 2005, respectively. Since July 2005, she has been an Assistant Professor in the Department of Electrical and Computer Engineering at the University of Victoria, British Columbia, Canada. Her research interests span several areas in wireless communications and networking, with a focus on network protocol and architecture design supporting emerging multimedia traffic over wireless, mobile, ad hoc, and sensor networks. She serves as the Associate Editor for IEEE Transactions on Vehicular Technology (2007- ), EURASIP Journal on Wireless Communications and Networking (2006- ), and International Journal of Sensor Networks (2006-). 